\title{
Non-Abelian Medium Effects in Quark-Gluon Plasma
}

\author{
Ji-sheng Chen ${ }^{a, b}, \quad$ Jia-rong $\mathrm{Li}^{b}, \quad$ Peng-fei Zhuang ${ }^{a}$ \\ ${ }^{a}$ Physics Department, Tsinghua University, Beijing 100084, P.R. China. \\ ${ }^{b}$ Institute of Particle Physics, Hua-Zhong Normal University, Wuhan 430079, P.R.China.
}

\begin{abstract}
Based on the kinetic theory, the non-Abelian medium property of hot Quark-Gluon Plasma is investigated. The nonlinearity of the plasma comes from two aspects: The nonlinear wave-wave interaction and self-interaction of color field. The non-Abelian color permittivity is obtained by expanding the kinetic equations to third order. As an application, the nonlinear Landau damping rate and the nonlinear eigenfrequency shift are calculated in the longwave length limit.
\end{abstract}

PACS:24.85.+p; 12.38.Mh; 51.10.+y; 77.22.Ch

\section{INTRODUCTION}

Looking for Quark-Gluon Plasma (QGP) as an important aim of relativistic heavy ion collisions has inspired wide interest and stimulated extensive studies $\mathrm{g}$. An urgent and important subject is to investigate medium effects in the hot plasma. The high transverse momentum particles and jets created by the hard scattering processes, which propagate through the hot and dense matter produced by the soft processes, can be used as a clear and observable probe of QGP 10.11 . Theoretically, to study the behavior of high energy partons through QGP one can construct a kind of response theory for QGP to external current, which must be non-Abelian and hence nonlinear 12. The non-Abelian permittivity, as a characteristic quantity of QGP medium, must be known.

The kinetic theory is a powerful tool to investigate the medium effects. The QGP kinetic theory which is fundamentally capable of dealing with the non-equilibrium phenomena has been formulated (see, for example, Ref. [1]), and the Abelian-like permittivity of QGP in the linear approximation has been given out 1 . However, in the linear approximation, the property of QGP is quite similar to QED plasma.

The great difference between the QED and QGP plasma is that the former is an Abelian medium while the latter is a non-Abelian one. It is not sufficient to take only the Abelian dominance approximation or the linear approximation in solving QGP kinetic equations. In order to reflect the essential characteristic of QGP, one must go beyond the linear approximation and take into account the nonlinear wave-wave interaction and the self-interaction of color field, as pointed out by Ref. [13].

In this paper, we aim at discussing the nonlinear medium effects in QGP based on the kinetic theory of chromodynamics. By using the collisionless Vlasov kinetic equations, the 
induced color currents up to third order are obtained. Then from the color field equation including both the nonlinear wave-wave interaction and the self-coupling term, the color permittivity of QGP is calculated. As an application, the nonlinear eigenfrequency shift and the nonlinear damping rate are calculated for the mode $\mathbf{k}=0$.

The paper is organized in the following. In Section II], we review the kinetic equations of QGP and discuss the expansion of the distribution function in the color mean field. Section III] is devoted to obtaining the first order, second order and third order color currents by solving the kinetic equations. In Section [V], the general expression of nonlinear permittivity is obtained by substituting the obtained color currents into the color field equation. The nonlinear Landau damping rate and the nonlinear eigenfrequency shift are obtained from the color permittivity in Section $\mathrm{V}$.

The natural units and the Minkovski metric are used through the paper, i.e., $\hbar=c=$ $k_{B}=1$ and $\operatorname{diag}\left(g_{\mu \nu}\right)=\operatorname{diag}\left(g^{\mu \nu}\right)=(1,-1,-1,-1)$.

\section{KINETIC EQUATIONS FOR FLUCTUATIONS}

\section{A. Mean Field and Kinetic Equations}

The color mean field equation in QGP is

$$
D_{\mu} F^{\mu \nu}(x)=j^{\nu}(x),
$$

with the color current

$$
j^{\nu}(x)=\frac{g}{2} \int \frac{d^{3} p}{(2 \pi)^{3} E}\left[\left(Q_{+}(x, p)-Q_{-}(x, p)\right)+2 i t_{a} f_{a b c} G_{b c}(x, p)\right]
$$

and the non-Abelian mean field stress tensor

$$
F_{\mu \nu}^{a}(x)=\partial_{\mu} A_{\nu}^{a}(x)-\partial_{\nu} A_{\mu}^{a}(x)+g f_{a b c} A_{\mu}^{b}(x) A_{\nu}^{c}(x) .
$$

Here, $t^{a}, a=1,2, \cdots, N_{c}^{2}-1$, are the $S U\left(N_{c}\right)$ group generators in fundamental representation and $f_{a b c}$ are the corresponding structure constants.

The quark and anti-quark distribution functions $Q_{ \pm}(x, p)$ are $N_{c} \times N_{c}$ matrices in color space, and behave as $Q(x, p) \rightarrow U(x, p) Q(x, p) U^{\dagger}(x, p)$ under the transformation operator $U(x, p)$. The Gluon distribution function $G(x, p)$ transforms as $G(x, p) \rightarrow$ $M(x, p) G(x, p) M^{\dagger}(x, p)$ with $M_{a b}=\operatorname{Tr}\left[t^{a} U(x, p) t^{b} U^{\dagger}(x, p)\right]$.

In the collisionless semi-classical limit with spin being neglected, the distribution functions $Q_{ \pm}(x, p)$ and $G(x, p)$ satisfy the following transport equations

$$
\begin{aligned}
p^{\mu} D_{\mu} Q_{ \pm}(x, p) \pm \frac{g}{2} p^{\mu} \frac{\partial}{\partial p_{\nu}}\left\{F_{\mu \nu}(x), Q_{ \pm}(x, p)\right\} & =0 \\
p^{\mu} \tilde{D}_{\mu} G(x, p)+\frac{g}{2} p^{\mu} \frac{\partial}{\partial p_{\nu}}\left\{\mathcal{F}_{\mu \nu}(x), G(x, p)\right\} & =0
\end{aligned}
$$

where $D_{\mu}$ and $\tilde{D}_{\mu}$ are the covariant derivatives defined as 


$$
\begin{aligned}
D_{\mu} & =\partial_{\mu}-i g\left[A_{\mu}, \cdots\right], \\
\tilde{D}_{\mu} & =\partial_{\mu}-i g\left[\mathcal{A}_{\mu}, \cdots\right] .
\end{aligned}
$$

The color mean field $A_{\mu}(x)$ and $\mathcal{A}_{\mu}$ and the field stress tensors $F_{\mu \nu}$ and $\mathcal{F}_{\mu \nu}$ have the following Lie-Algebra relations

$$
\begin{aligned}
& A^{\mu}(x)=A_{a}^{\mu}(x) t^{a}, \quad F^{\mu \nu}(x)=F_{a}^{\mu \nu}(x) t^{a}, \\
& \mathcal{A}_{a b}(x)=-i f_{a b c} A_{c}^{\mu}(x), \quad \mathcal{F}_{a b}^{\mu \nu}(x)=-i f_{a b c} F_{c}^{\mu \nu}(x) .
\end{aligned}
$$

The Eqs.(2.1) and (2.4) form a closed set of coupled equations. It is easy to see that the nonlinearity in QGP results from two aspects. One is associated with the nonlinear kinetic equations (2.4), and the other one is related to the self-interaction term on the left hand side of mean field equation (2.1).

An usually used approximation to solve the above coupled equations is the linearization. Linearizing the left hand side of mean field equation (2.4), i.e., neglecting the self-interaction term, was considered in Refs. [14,15], and linearizing the kinetic equations was discussed by Blaizot et al. in Ref. [3, [16]. What we will do in this paper is to discuss the nonlinear effects in both the field and kinetic equations.

\section{B. Equations of Fluctuations}

For convenience to analyze the wave-wave interaction of fluctuations in QGP, the mean fields and the distribution functions can be divided into regular parts and fluctuation parts 5.,13,14.21. The quark, anti-quark and gluon distribution functions are decomposed as

$$
\begin{aligned}
& Q_{ \pm}(x, p)=\bar{Q}_{ \pm}(x, p)+q_{ \pm}(x, p), \\
& G(x, p)=\bar{G}(x, p)+g(x, p),
\end{aligned}
$$

and in the meantime, the field $A$ and current $j^{\mu}$ are decomposed as

$$
A_{\mu}^{a}(x)=\bar{A}_{\mu}^{a}(x)+A_{\mu}^{a T}(x), \quad j_{a}^{\mu}(x)=\bar{j}_{\mu}^{a}(x)+j_{\mu}^{a T}(x) .
$$

Here the quantities having a bar represent the ensemble average of the corresponding quantities, and by definition the mean values of the fluctuations vanish

$$
\begin{aligned}
& \left\langle q_{ \pm}(x)\right\rangle=0, \quad\langle g(x)\rangle=0, \\
& \left\langle A^{T}(x)\right\rangle=0, \quad\left\langle j^{T}(x)\right\rangle=0 .
\end{aligned}
$$

With the above decomposition, one can obtain the kinetic equations for the fluctuations

of distribution functions by subtracting the kinetic equations for the regular distributions from the average of the kinetic equations (2.4)

$$
\begin{gathered}
p^{\mu} \partial_{\mu} q_{ \pm}(x, p)=i g\left(\left[A_{\mu}^{T}(x), q_{ \pm}(x, p)\right]-\left\langle\left[A_{\mu}^{T}(x), q_{ \pm}(x, p)\right]\right\rangle\right) \mp \frac{1}{2} g p^{\mu}\left\{F_{\mu \nu L}^{T}(x), \frac{\partial \bar{Q}_{ \pm}(x, p)}{\partial p_{\nu}}\right\} \\
\mp \frac{1}{2} g p^{\mu}\left(\left\{F_{\mu \nu L}^{T}(x), \frac{\partial q_{ \pm}(x, p)}{\partial p_{\nu}}\right\}-\left\langle\left\{F_{\mu \nu L}^{T}(x), \frac{\partial q_{ \pm}(x, p)}{\partial p_{\nu}}\right\}\right\rangle\right)
\end{gathered}
$$




$$
\begin{aligned}
& \quad \frac{1}{2} i g^{2} p^{\mu}\left(\left\{\left[A_{\mu}^{T}(x), A_{\nu}^{T}(x)\right], \frac{\partial q_{ \pm}(x, p)}{\partial p_{\nu}}\right\}-\left\langle\left\{\left[A_{\mu}^{T}(x), A_{\nu}^{T}(x)\right], \frac{\partial q_{ \pm}(x, p)}{\partial p_{\nu}}\right\}\right\rangle\right) \\
& \quad \pm \frac{1}{2} i g^{2} p^{\mu}\left\{\left[A_{\mu}^{T}(x), A_{\nu}^{T}(x)\right]-\left\langle\left[A_{\mu}^{T}(x), A_{\nu}^{T}(x)\right]\right\rangle, \frac{\partial \bar{Q}_{ \pm}(x, p)}{\partial p_{\nu}}\right\}, \\
& p^{\mu} \partial_{\mu} g(x, p)=i g\left(\left[\mathcal{A}_{\mu}^{T}(x), g(x, p)\right]-\left\langle\left[\mathcal{A}_{\mu}^{T}(x), g(x, p)\right]\right\rangle\right)-\frac{1}{2} g p^{\mu}\left\{\mathcal{F}_{\mu \nu L}^{T}(x), \frac{\partial \bar{G}(x, p)}{\partial p_{\nu}}\right\} \\
& \quad-\frac{1}{2} g p^{\mu}\left(\left\{\mathcal{F}_{\mu \nu L}^{T}(x), \frac{\partial g(x, p)}{\partial p_{\nu}}\right\}-\left\langle\left\{\mathcal{F}_{\mu \nu L}^{T}(x), \frac{\partial g(x, p)}{\partial p_{\nu}}\right\}\right\rangle\right) \\
& \quad+\frac{1}{2} i g^{2} p^{\mu}\left(\left\{\left[\mathcal{A}_{\mu}^{T}(x), \mathcal{A}_{\nu}^{T}(x)\right], \frac{\partial g(x, p)}{\partial p_{\nu}}\right\}-\left\langle\left\{\left[\mathcal{A}_{\mu}^{T}(x), \mathcal{A}_{\nu}^{T}(x)\right], \frac{\partial g(x, p)}{\partial p_{\nu}}\right\}\right\rangle\right) \\
& \quad+\frac{1}{2} i g^{2} p^{\mu}\left\{\left[\mathcal{A}_{\mu}^{T}(x), \mathcal{A}_{\nu}^{T}(x)\right]-\left\langle\left[\mathcal{A}_{\mu}^{T}(x), \mathcal{A}_{\nu}^{T}(x)\right]\right\rangle, \frac{\partial \bar{G}(x, p)}{\partial p_{\nu}}\right\},
\end{aligned}
$$

where the index $L$ denote the linear term of $F_{\mu \nu}$ in $A_{\mu}^{T}$.

The fluctuation part of the color field satisfies the following Yang-Mills equation

$$
\begin{aligned}
& \partial_{\mu} F_{L}^{T \mu \nu}+j^{T(1) \nu}=-j_{N L}^{T \nu}+i g \partial_{\mu}\left(\left[A^{T \mu}, A^{T \nu}\right]-\left\langle\left[A^{T \mu}, A^{T \nu}\right]\right\rangle\right) \\
& \quad+i g\left(\left[A_{\mu}^{T}, F_{L}^{T \mu \nu}\right]-\left\langle\left[A_{\mu}^{T}, F_{L}^{T \mu \nu}\right]\right\rangle\right)+g^{2}\left(\left[A_{\mu}^{T},\left[A^{T \mu}, A^{T \nu}\right]\right]-\left\langle\left[A_{\mu}^{T},\left[A^{T \mu}, A^{T \nu}\right]\right]\right\rangle\right),
\end{aligned}
$$

where the current fluctuation $j^{T \mu}$ is also divided into a linear term $j^{T(1) \nu}$ and a nonlinear term $j_{N L}^{T \nu}$. By expanding the fluctuation of the distribution functions in terms of the fluctuations of the color field $A_{\mu}^{a T}$

$$
\begin{gathered}
q_{ \pm}(x, p)=\sum_{n=1}^{\infty} q_{ \pm}^{(n)}(x, p), \\
g(x, p)=\sum_{n=1}^{\infty} g^{(n)}(x, p),
\end{gathered}
$$

the nonlinear color current can be expressed as

$$
j^{T \mu}=\sum_{n=2}^{\infty} j^{T(n) \mu},
$$

with

$$
\begin{array}{r}
j^{T(n) \mu}(x)=g t^{a} \int \frac{d^{3} p}{(2 \pi)^{3} p^{0}} p^{\mu}\left[\operatorname{tr}\left(t^{a}\left\{q_{+}^{(n)}(x, p)-q_{-}^{(n)}(x, p)\right\}\right)+\operatorname{Tr}\left(T^{a} g^{(n)}(x, p)\right)\right], \\
n=2,3,4, \cdots .
\end{array}
$$

Substituting the above expansion of fluctuations in $A_{\mu}^{T}$ into the kinetic equations (2.10), one obtains the hierarchies of kinetic equations for the distribution functions $q_{ \pm}^{(n)}$ and $g^{(n)}$.

By using the regular distributions and regular field as input, which can be calculated from the corresponding kinetic equations and mean field equation, one can in principle solve the above kinetic and mean field equations for the fluctuations 1.62122 . For simplification, we will consider in the following the fluctuations above the colorless equilibrium state. We take $\bar{A}=0$ and Fermi-Dirac or Bose-Einstein distribution function for $\bar{Q}_{ \pm}$and $\bar{G}$ 


$$
\bar{Q}_{ \pm}=\frac{2 N_{f}}{e^{\beta p \cdot u}+1}, \quad \bar{G}=\frac{2}{e^{\beta p \cdot u}-1}
$$

where $u=(1,0,0,0)$ is the local four-velocity of the plasma, $N_{f}$ is the the number of flavors

for quarks. Since we are interested in the fluctuations only, the index $T$ for the field and current will be omitted from now on.

It's convenient to discuss the fluctuations in momentum space through the Fourier transformation,

$$
q_{ \pm}(x, p)=\int \frac{d^{4} k}{(2 \pi)^{4}} q_{ \pm}(k, p) e^{-i k x}
$$

We choose temporary axis gauge, i.e., $A_{0}=0$, and consider only the longitudinal field excitation, $A^{i}(k)=\frac{k^{i} A(k)}{K}$ with $K=|\mathbf{k}|$.

\section{LINEAR AND NONLINEAR COLOR CURRENTS}

\section{A. Linear Approximation}

In order to investigate the nonlinear property of QGP, one should solve the kinetic hierarchies for the distributions $q_{ \pm}^{(n)}$ and $g^{(n)}$ order by order. Let's first review the linear approximation which has been discussed in many previous works. The dispersion relation obtained here will be used to perform the relevant integrals in the end of this paper.

¿From the kinetic equations for the first order fluctuations of the distribution functions, it is easy to write down

$$
\begin{aligned}
& q_{ \pm}^{(1)}(k, p)=\mp \frac{k^{0} p^{i} A^{i}(k)}{p \cdot k+i p^{0} \epsilon^{+}} \cdot \frac{\partial \bar{Q}_{ \pm}\left(p^{0}\right)}{\partial p^{0}} \\
& g^{(1)}(k, p)=-\frac{k^{0} p^{i} \mathcal{A}^{i}(k)}{p \cdot k+i p^{0} \epsilon^{+}} \cdot \frac{\partial \bar{G}\left(p^{0}\right)}{\partial p^{0}} .
\end{aligned}
$$

Substituting these first order fluctuations into (2.14), one obtains the first order color current by noting $\operatorname{Tr} T^{a} T^{b}=N_{c} \delta^{a b}$. Then from the mean field equation to the first order, one has the nontrivial solution

$$
-\omega^{2} \varepsilon_{L}(\omega, \mathbf{k}) A(k)=0
$$

with the well-known linear dispersion relation

$$
\varepsilon_{L}(\omega, \mathbf{k})=1+\frac{3 \omega_{p}^{2}}{K^{2}}\left[1-\frac{\omega}{2 K}\left(\ln \left|\frac{K+\omega}{K-\omega}\right|-i \pi \theta(K-\omega)\right)\right]=0
$$

where

$$
\omega_{p}=\sqrt{\frac{\left(N_{f}+2 N_{c}\right)}{18} g^{2} T^{2}}
$$


is the plasma frequency. In the longwave length region, the dispersion relation is reduced to

$$
\omega^{2}=\omega_{p}^{2}+\frac{3}{5} k^{2} .
$$

It is necessary to note that the linear dispersion relation (3.3) means that the eigenwaves in QGP are always timelike, they can not exchange energy directly with the particles in the plasma. Therefore in the linear approximation, the eigenwaves in QGP do not undergo damping

To obtain the nonlinear effects in the medium, one should solve the kinetic equations to nonlinear orders, i.e., to go beyond the linear order approximation.

\section{B. Second Order and Third Order Color Currents}

In this subsection, the second order and third order color currents will be calculated. The following shorthands

$$
\begin{aligned}
\sum_{k=k_{1}+k_{2}} & =\int \delta\left(k-k_{1}-k_{2}\right) d k_{1} d k_{2} \frac{1}{(2 \pi)^{4}}, \\
\sum_{k=k_{1}+k_{2}+k_{3}} & =\int \delta\left(k-k_{1}-k_{2}-k_{3}\right) d k_{1} d k_{2} d k_{3} \frac{1}{(2 \pi)^{8}},
\end{aligned}
$$

are used for notational simplification.

The second order kinetic equation for the quark distribution is the following

$$
\begin{aligned}
-i p^{\mu} k_{\mu} q_{+}^{(2)}(k, p)=i g p^{i} \sum_{k_{1}+k_{2}=k}\left(\left[A_{i}\left(k_{1}\right), q_{+}^{(1)}\left(k_{2}, p\right)\right]-\left\langle\left[A_{i}\left(k_{1}, p\right), q_{+}^{(1)}\left(k_{2}, p\right)\right]\right\rangle\right) \\
+\frac{1}{2} g p^{\mu} \sum_{k_{1}+k_{2}=k}\left(k_{1 \mu}\left\{A_{i}\left(k_{1}\right), \frac{\partial q_{+}^{(1)}\left(k_{2}, p\right)}{\partial p_{i}}\right\}-\left\langle k_{1 \mu}\left\{A_{i}\left(k_{1}\right), \frac{\partial q_{+}^{(1)}\left(k_{2}, p\right)}{\partial p_{i}}\right\}\right\rangle\right) \\
\quad-\frac{i}{2} g p^{i} \sum_{k=k_{1}+k_{2}}\left(\left\{k_{1 \nu} A_{i}\left(k_{1}\right), \frac{\partial q_{+}^{(1)}\left(k_{2}, p\right)}{\partial p_{\nu}}\right\}-\left\langle\left\{k_{1 \nu} A_{i}\left(k_{1}\right), \frac{\partial q_{+}^{(1)}\left(k_{2}, p\right)}{\partial p_{\nu}}\right\}\right\rangle\right) \\
\quad+\frac{i}{2} g p^{i} \sum_{k=k_{1}+k_{2}+k_{3}}\left(\left\{\left[A_{i}\left(k_{1}\right), A_{j}\left(k_{2}\right)\right], \frac{\partial \bar{Q}_{+}\left(k_{3}, p\right)}{\partial p_{j}}\right\}-\left\langle\left\{\left[A_{i}\left(k_{1}\right), A_{j}\left(k_{2}\right)\right], \frac{\partial \bar{Q}_{+}\left(k_{3}, p\right)}{\partial p_{j}}\right\}\right\rangle\right) .
\end{aligned}
$$

The kinetic equations for the second order fluctuations of the anti-quark and gluon distribution functions can be written out similarly to (3.8) with opposite signs for the terms related to $\{\cdots\}$ for anti-quarks and with the replacement of $q, A$ and $F_{\mu \nu}$ by $G, \mathcal{A}$ and $\mathcal{F}_{\mu \nu}$ for gluons.

Inserting the second order distribution functions into Eq.(2.14), one obtains the second order color current

$$
\begin{aligned}
& j^{T(2) k a}= \\
& -i g^{3} f_{a b c} \sum_{k=k_{1}+k_{2}} \int \frac{d^{3} p}{(2 \pi)^{3} p^{0}} \frac{p^{i} p^{j} p^{k}}{p \cdot k+i p^{0} \varepsilon} \cdot \frac{\omega_{2} \partial_{p_{0}} \mathcal{D}_{e q}}{p \cdot k_{2}+i p^{0} \varepsilon}\left(A_{i}^{b}\left(k_{1}\right) A_{j}^{c}\left(k_{2}\right)-\left\langle A_{i}^{b}\left(k_{1}\right) A_{j}^{c}\left(k_{2}\right)\right\rangle\right),
\end{aligned}
$$


with the effective equilibrium distribution function

$$
\mathcal{D}_{e q}=\frac{1}{2} N_{f}\left(\bar{Q}_{+}+\bar{Q}_{-}\right)+N_{c} \bar{G} .
$$

The third order color current can be obtained similarly

$$
\begin{aligned}
& j^{T(3) l a}(k)= \\
& \sum_{k=k_{1}+k_{2}+k_{3}} \sum_{k k_{1} k_{2} k_{3}}^{a b d e i j k l}\left(A_{i}^{b}\left(k_{3}\right) A_{j}^{d}\left(k_{1}\right) A_{k}^{e}\left(k_{2}\right)-A_{i}^{b}\left(k_{3}\right)\left\langle A_{j}^{d}\left(k_{1}\right) A_{k}^{e}\left(k_{2}\right)\right\rangle-\left\langle A_{i}^{b}\left(k_{3}\right) A_{j}^{d}\left(k_{1}\right) A_{k}^{e}\left(k_{2}\right)\right\rangle\right),
\end{aligned}
$$

with

$$
\begin{aligned}
\Sigma_{k k_{1} k_{2} k_{3}}^{a b d e i k l}= & f^{a b c} f^{c d e} \Sigma_{k k_{1} k_{2} k_{3}}^{(I) i j k l}+\delta^{a b} \delta^{d e} \Sigma_{k k_{1} k_{2} k_{3}}^{(I I) i j k l}+d^{a b c} d^{c d e} \Sigma_{k k_{1} k_{2} k_{3}}^{(I I I) i j k l} \\
& +\left(\delta^{a b} \delta^{d e}+\delta^{a d} \delta^{b e}+\delta^{a e} \delta^{d b}\right) \Sigma_{k k_{1} k_{2} k_{3}}^{(V I) i j k l}, \\
\Sigma_{k k_{1} k_{2} k_{3}}^{(I) i j k l}= & -g^{4} \int \frac{d^{3} p}{(2 \pi)^{3} p^{0}} \frac{p^{i} p^{j} p^{k} p^{l}}{p \cdot k+i p^{0} \epsilon} \frac{1}{p \cdot\left(k_{1}+k_{2}\right)+i p^{0} \epsilon} \frac{\omega_{p_{0}} \mathcal{D}_{e q}}{p \cdot k_{2}+i p^{0} \epsilon}, \\
\Sigma_{k k_{1} k_{2} k_{3}}^{(I I) i j k l}= & \frac{2}{N_{c}} \Sigma_{k k_{1} k_{2} k_{3}}^{(I I I) i j k l} \\
= & -\frac{g^{4}}{2 N_{c}} \int \frac{d^{3} p}{(2 \pi)^{3} p^{0}} \frac{p^{l} \chi^{i \mu}\left(k_{3}, p\right)}{p \cdot k+i p^{0} \epsilon} \frac{\partial}{\partial p^{\mu}}\left(\frac{\chi^{j \nu}\left(k_{1}, p\right)}{p \cdot\left(k_{1}+k_{2}\right)+i p^{0} \epsilon} \frac{\partial}{\partial p^{\nu}}\left(\frac{\omega_{2} \partial_{p}^{0} \mathcal{D}_{e q}}{p \cdot k_{2}+i p^{0} \epsilon}\right)\right), \\
\Sigma_{k k_{1} k_{2} k_{3}}^{(V I) i j k l}= & -\frac{g^{4}}{2} \int \frac{d^{3} p}{(2 \pi)^{3} p^{0}} \frac{p^{l} \chi^{i \mu}\left(k_{3}, p\right)}{p \cdot k+i p^{0} \epsilon} \frac{\partial}{\partial p^{\mu}}\left(\frac{\chi^{j \nu}\left(k_{1}, p\right)}{p \cdot\left(k_{1}+k_{2}\right)+i p^{0} \epsilon} \frac{\partial}{\partial p^{\nu}}\left(\frac{\omega_{2} \partial_{p}^{0} \bar{G}}{p \cdot k_{2}+i p^{0} \epsilon}\right)\right),
\end{aligned}
$$

and

$$
\chi^{\mu \nu}(k, p)=(p \cdot k) g^{\mu \nu}-p^{\mu} k^{\nu}
$$

where $d_{b c e}$ are the $S U\left(N_{c}\right)$ symmetric structure constants. From Eqs.(3.9) and (3.11), one can see that the second and third order color currents come from the response of particles to the secondary waves resulting from the nonlinear interaction of eigenwaves.

Before making further calculations, one should note that on the right hand side of (3.11), the term with $\Sigma_{k k_{1} k_{2} k_{3}}^{(I) i j k}$ is purely non-Abelian, while the others are Abelian terms. Since the soft excitation momentum $k \sim g T$ and the particle momentum $p \sim T$, 3 , one can easily conclude that the leading non-Abelian term is proportional to $g^{2}$, while the Abelian terms are proportional to the higher orders of the coupling constant $g: \Sigma_{k k_{1} k_{2} k_{3}}^{(I I) i j k l} \sim \Sigma_{k k_{1} k_{2} k_{3}}^{(I I I) i k l} \sim g^{3}$ and $g^{4}$ and $\Sigma_{k k_{1} k_{2} k_{3}}^{(V I) i j k l} \sim g^{3}$.

\section{NONLINEAR PERMITTIVITY}

Now we turn to calculate the non-Abelian permittivity from the mean field equation to the third order

$$
\begin{aligned}
& -k_{\mu} k^{\mu} A^{i}(k)+k_{j} k^{i} A^{j}(k)+j^{T(1) i}(k)= \\
& g^{2} \sum_{k=k_{1}+k_{2}+k_{3}}\left(\left[A_{j}\left(k_{1}\right),\left[A^{j}\left(k_{2}\right), A^{i}\left(k_{3}\right)\right]\right]-\left\langle\left[A_{j}\left(k_{1}\right),\left[A^{j}\left(k_{2}\right), A^{i}\left(k_{3}\right)\right]\right]\right\rangle\right)-j_{N L}^{T i}(k),
\end{aligned}
$$


with

$$
j_{N L}^{T i}(k)=j^{T(2) i}(k)+j^{T(3) i}(k)
$$

¿From (4.1), one can see that the left hand side is Abelian-like, while the right hand side is non-Abelian which results from two different origins. The first term on the right hand side of (4.1) with the summation represents the color self-interaction. Some of our previous works 14,15] neglected this nonlinearity. The second non-Abelian property in (4.1) is from the nonlinear current $j_{\mu}^{T i}$. This nonlinear wave-wave interaction is not taken into account in Refs. [3, 4, 17]. Here we will discuss the solution of the field equation by considering the color self-interaction and the nonlinear currents simultaneously.

Substituting $j^{T(2)}(k)$ and $j^{T(3)}(k)$ into the field equation (4.1), one gets the relation

$$
\begin{aligned}
& -\omega^{2} A(k)+\mathbf{j}^{T(1)} \cdot \frac{\mathbf{k}}{K}= \\
& i g^{3} t^{a} f^{a b c} \sum_{k=k_{1}+k_{2}} \int \frac{d^{3} p}{(2 \pi)^{3} p^{0}} \frac{(\mathbf{p} \cdot \mathbf{k})}{K} \frac{\left(\mathbf{p} \cdot \mathbf{k}_{\mathbf{1}}\right)}{K_{1}} \frac{\left(\mathbf{p} \cdot \mathbf{k}_{\mathbf{2}}\right)}{K_{2}} \frac{1}{p \cdot k+i p^{0} 0^{+}} \frac{\omega_{2} \frac{\partial \mathcal{D}_{e q}}{\partial p_{0}}}{p \cdot k_{2}+i p^{0} 0^{+}} \\
& \quad \times\left(A^{b}\left(k_{1}\right) A^{c}\left(k_{2}\right)-\left\langle A^{b}\left(k_{1}\right) A^{c}\left(k_{2}\right)\right\rangle\right) \\
& -g^{2} t^{a} \sum_{k=k_{1}+k_{2}+k_{3}}\left[g^{2} \int \frac{d^{3} p}{(2 \pi)^{3} p^{0}} \frac{\left(\mathbf{p} \cdot \mathbf{k}_{\mathbf{1}}\right)\left(\mathbf{p} \cdot \mathbf{k}_{\mathbf{2}}\right)\left(\mathbf{p} \cdot \mathbf{k}_{\mathbf{3}}\right)(\mathbf{p} \cdot \mathbf{k})}{K_{1} K_{2} K_{3} K} \frac{1}{p \cdot k+i p^{0} \epsilon}\right. \\
& \left.\quad \times \frac{1}{p \cdot\left(k_{1}+k_{2}\right)+i p^{0} \epsilon} \frac{\omega_{2} \partial_{p_{0}} \mathcal{D}_{e q}}{p \cdot k_{2}+i p^{0} \epsilon}+\frac{\left(\mathbf{k}_{1} \cdot \mathbf{k}_{2}\right)}{K_{1} K_{2}} \frac{\left(\mathbf{k} \cdot \mathbf{k}_{3}\right)}{K K_{3}}\right] \\
& \quad \times f^{a b c} f^{c d e}\left(A^{b}\left(k_{3}\right) A^{d}\left(k_{1}\right) A^{e}\left(k_{2}\right)-A^{b}\left(k_{3}\right)\left\langle A^{d}\left(k_{1}\right) A^{e}\left(k_{2}\right)\right\rangle-\left\langle A^{b}\left(k_{3}\right) A^{d}\left(k_{1}\right) A^{e}\left(k_{2}\right)\right\rangle\right) .
\end{aligned}
$$

Obviously the first and second integrals reflect the three-wave process $\left(k \rightleftharpoons k_{1}+k_{2}\right)$ and the four-wave $\left(k \rightleftharpoons k_{1}+k_{2}+k_{3}\right)$ process, respectively.

It is well known that it is difficult to solve the general Yang-Mills equation because of the color field self-coupling term. However, if the phase of excitations in QGP is random (Gauss-like distributed), the correlation of even random quantities can be approximately expressed as the product of correlations of two quantities 21,23, and the solution of the field equation can be greatly simplified. Concretely, after multiplying both sides of (4.3) with $A^{g}\left(k^{\prime}\right)$, taking average with respect to the Gauss-like random phase, and then using the following relations 23

$$
\begin{aligned}
I_{k}^{d g}=\left\langle A^{d} A^{g}\right\rangle_{\omega, \mathbf{k}}, \quad & \left\langle A^{d}(k) A^{g}\left(k^{\prime}\right)\right\rangle=(2 \pi)^{4} I_{k}^{d g} \delta\left(k+k^{\prime}\right), \\
\left\langle A^{a}\left(k_{1}\right) A^{b}\left(k_{2}\right) A^{c}\left(k_{3}\right) A^{d}\left(k_{4}\right)\right\rangle= & (2 \pi)^{8} \delta\left(k_{1}+k_{2}+k_{3}+k_{4}\right) \times\left(\delta\left(k_{1}+k_{2}\right) I_{k_{1}}^{a b} I_{k_{3}}^{c d}\right. \\
& \left.+\delta\left(k_{1}+k_{3}\right) I_{k_{1}}^{a c} I_{k_{2}}^{b d}+\delta\left(k_{1}+k_{4}\right) I_{k_{1}}^{a d} I_{k_{2}}^{b c}\right),
\end{aligned}
$$

the equation (4.3) is reduced to

$$
\left(\varepsilon_{L} \delta_{a d}+\varepsilon_{a d}^{*}\right) I_{k}^{d g}=0,
$$

where $\varepsilon_{L}$ is the linear permittivity given in (3.3), and the nonlinear permittivity $\varepsilon_{a d}^{*}$ contains two parts 


$$
\varepsilon_{a d}^{*}=\varepsilon_{a d}^{R *}+\varepsilon_{a d}^{S *},
$$

with

$$
\begin{aligned}
\varepsilon_{a d}^{S *}= & -\frac{g^{2}}{\omega^{2}} \int\left[f^{a b c} f^{c e d} I_{k_{1}}^{b e}+\left(\frac{\mathbf{k} \cdot \mathbf{k}_{1}}{K K_{1}}\right)^{2}\left(f^{a b c} f^{c d e} I_{k_{1}}^{b e}+f^{a d c} f^{c b e} I_{k_{1}}^{b e}\right)\right] \frac{d k_{1}}{(2 \pi)^{4}}, \\
\varepsilon_{a d}^{R *}= & \frac{g^{4}}{\omega^{2}} \int \frac{d^{3} p \partial_{p_{0}} \mathcal{D}_{e q}}{(2 \pi)^{3} p^{0}} f^{a b c}\left(\frac{\mathbf{p} \cdot \mathbf{k}_{1}}{K_{1}}\right)^{2}\left(\frac{\mathbf{p} \cdot \mathbf{k}}{K}\right)^{2} \frac{1}{p \cdot k+i p^{0} \epsilon} \\
& \times \frac{1}{p \cdot\left(k-k_{1}\right)+i p^{0} \epsilon}\left(\frac{\omega}{p \cdot k+i p^{0} \epsilon} f^{c e d} I_{k_{1}}^{b e}+\frac{\omega_{1}}{p \cdot k_{1}+i p^{0} \epsilon} f^{c d e} I_{k_{1}}^{b e}\right) \frac{d k_{1}}{(2 \pi)^{4}} .
\end{aligned}
$$

Here $\varepsilon_{a d}^{S *}$ and $\varepsilon_{a d}^{R *}$ correspond to the first and second term on the right hand side of (4.1), respectively. They reflect the purely non-Abelian medium effects of QGP. From Eq. (4.7), the nonlinear permittivity $\varepsilon_{a d}^{*}$ is a matrix in color space because of the non-Abelian $S U\left(N_{c}\right)$ structure constants. Its value depends on the correlation strength $I_{k}^{b c}=\left\langle A^{b} A^{c}\right\rangle_{k}$ and the dispersion relation $\omega(\mathbf{k})$ of the collective excitation.

For simplification, we consider only the correlation between the same colors and take a diagonal matrix in the color space

$$
\left\langle A^{b} A^{c}\right\rangle_{k}=-\frac{\pi}{\omega^{2}}\left(\delta\left(\omega-\omega_{\mathbf{k}}\right)+\delta\left(\omega+\omega_{\mathbf{k}}\right)\right) I_{\mathbf{k}} \delta_{b c}
$$

where $I_{\mathbf{k}}$ is the intensity of the correlation with frequency $\omega_{\mathbf{k}}$. For fluctuations at thermal level, one can take $I_{\mathbf{k}}=4 \pi T$ 13:25]. Then the nonlinear contributions $\varepsilon_{a d}^{S *}$ and $\varepsilon_{a d}^{R *}$ to the color permittivity are further reduced to

$$
\begin{aligned}
\varepsilon^{S *}= & -\frac{2 g^{2} N_{c}}{\omega^{2}(2 \pi)^{4}} \int\left(1-\left(\frac{\mathbf{k} \cdot \mathbf{k}_{1}}{K K_{1}}\right)^{2}\right) \frac{\pi I_{\mathbf{k}_{1}}}{\omega_{\mathbf{k}_{1}}^{2}} d^{3} k_{1} \\
\varepsilon^{R *}= & \frac{2 g^{4} N_{c}}{\omega^{2}} \int \frac{d^{3} p \partial_{p}^{0} \mathcal{D}_{e q}}{(2 \pi)^{7} p^{0}}\left(\frac{\mathbf{p} \cdot \mathbf{k}_{1}}{K_{1}}\right)^{2}\left(\frac{\mathbf{p} \cdot \mathbf{k}}{K}\right)^{2} \frac{1}{p \cdot k+i p^{0} \epsilon} \\
& \times\left(\frac{1}{p \cdot\left(k-k_{1}\right)+i p^{0} \epsilon} \frac{\omega_{0}}{p \cdot k+i p^{0} \epsilon}-\frac{1}{p \cdot\left(k+k_{1}\right)+i p^{0} \epsilon} \frac{\omega_{\mathbf{k}_{1}}}{p \cdot k_{1}+i p^{0} \epsilon}\right) \frac{\pi I_{\mathbf{k}_{1}}}{\omega_{\mathbf{k}_{1}}^{2}} d^{3} k_{1} .
\end{aligned}
$$

The four factors $\frac{1}{p \cdot k+i p^{0} \epsilon}, \frac{1}{p \cdot k_{1}+i p^{0} \epsilon}, \frac{1}{p \cdot\left(k+k_{1}\right)+i p^{0} \epsilon}, \frac{1}{p \cdot\left(k-k_{1}\right)+i p^{0} \epsilon}$ in the expression of $\varepsilon^{R *}$ have different contributions to the integral. The first three factors have only real contributions because the eigenwaves are always timelike, while the last one has both real and imaginary contributions to $\varepsilon^{R}$ due to the well-know formula

$$
\frac{1}{p \cdot\left(k-k_{1}\right)+i p^{0} \epsilon}=P \frac{1}{p \cdot\left(k-k_{1}\right)}-i \frac{\pi}{p^{0}} \delta\left(\omega_{\mathbf{k}}-\omega_{\mathbf{k}_{1}}-\mathbf{v} \cdot\left(\mathbf{k}-\mathbf{k}_{1}\right)\right),
$$

where $\mathbf{v}=\mathbf{p} / p^{0}$. The imaginary part of $\varepsilon^{R *}$ indicates that the secondary waves resulting from the nonlinear interactions can exchange energy with the plasma particles, which corresponds to the nonlinear Landau damping.

To perform the above integrals over $\mathbf{k}_{1}$ and $\mathbf{p}$, we can choose spherical polar coordinates and take the polar axis of $\mathbf{k}_{1}$ in the direction of $\mathbf{k}$ and the polar axis of $\mathbf{p}$ in the direction of 
$\mathbf{k}_{1}$. Considering the soft excitation in QGP 6 , the up and low limits of $K_{1}$ can be chosen to be $g T$ and $g^{2} T$, respectively.

By using Eq. (4.10) and the integral

$$
-\int_{0}^{\infty} p_{0}^{2} \partial_{p_{0}} \mathcal{D}_{e q} d p_{0}=\frac{\left(N_{f}+2 N_{c}\right) \pi^{2} T^{2}}{3}
$$

the nonlinear color permittivities $\varepsilon^{S *}$ and $\varepsilon^{R *}$ are finally written as one dimensional integrals

$$
\begin{aligned}
\varepsilon^{S *}= & -\frac{64 \pi^{3} g^{2} N_{c}}{3(2 \pi)^{4} \omega_{p}^{2}} \int_{g^{2} T}^{g T} \frac{1}{\omega_{\mathbf{k}_{1}}^{2}} K_{1}^{2} d K_{1}, \\
\operatorname{Re}\left(\varepsilon^{R *}\right)= & \\
- & \frac{8 g^{4} N_{c} \pi^{3} T^{3}\left(N_{f}+2 N_{c}\right)}{9(2 \pi)^{4} \omega_{p}^{3}}\left[\int_{g^{2} T}^{g T} \frac{K_{1}^{2} d K_{1}}{\omega_{\mathbf{k}_{1}}^{2}} \frac{2\left(\omega_{\mathbf{k}_{1}}-\omega_{p}\right)}{K_{1}^{2}}\left(1-\frac{\omega_{\mathbf{k}_{1}}-\omega_{p}}{2 K_{1}} \ln \left|\frac{\omega_{p}-\omega_{\mathbf{k}_{1}}-K_{1}}{\omega_{p}-\omega_{\mathbf{k}_{1}}+K_{1}}\right|\right)\right. \\
& \left.-\int_{g^{2} T}^{g T} \frac{K_{1}^{2} d K_{1}}{\omega_{\mathbf{k}_{1}}} \frac{2}{K_{1}^{2}}\left(1+\frac{\omega_{\mathbf{k}_{1}}^{2}}{2 \omega_{p} K_{1}} \ln \left|\frac{\omega_{\mathbf{k}_{1}}-K_{1}}{\omega_{\mathbf{k}_{1}}+K_{1}}\right|-\frac{\left(\omega_{\mathbf{k}_{1}}-\omega_{p}\right)^{2}}{2 \omega_{p} K_{1}} \ln \left|\frac{\omega_{\mathbf{k}_{1}}-\omega_{p}-K_{1}}{\omega_{\mathbf{k}_{1}}-\omega_{p}+K_{1}}\right|\right)\right], \\
\operatorname{Im}\left(\varepsilon^{R *}\right)= & \frac{8 g^{4} N_{c} \pi^{4} T^{3}\left(N_{f}+2 N_{c}\right)}{9(2 \pi)^{4} \omega_{p}^{4}} \int_{g^{2} T}^{g T} \frac{1}{K_{1}} d K_{1}\left(\omega_{\mathbf{k}_{1}}-\omega_{p}\right)^{2} \frac{1}{\omega_{\mathbf{k}_{1}}} .
\end{aligned}
$$

The contributions of $\varepsilon^{R *}$ and $\varepsilon^{S *}$ can be seen quantitatively by making a rough numerical estimate. Using the dispersion relation (3.5) in the longwave length limit, the total nonlinear permittivity is

$$
\varepsilon^{*}=\varepsilon^{S *}+\operatorname{Re}\left(\varepsilon^{R *}\right)+i \operatorname{Im}\left(\varepsilon^{R *}\right) \approx-0.98 g-1.91 g+0.61 g i=-2.89 g+0.61 g i .
$$

\section{CONCLUSION}

Based on the chromodynamic kinetic and mean field equations, the nonlinearity in QGP has been analyzed. One nonlinearity comes from the response of particles to the secondary waves resulting from the nonlinear interaction, and the other nonlinearity is due to the nonAbelian self-interaction in mean field equation. The non-Abelian permittivity due to the two nonlinearities has been calculated by considering the second and third order color currents. ¿From its general expression, it depends on the dispersion modes $\omega_{\mathbf{k}}$ and the correlation strength of color field. The non-Abelian property is reflected in the presence of the $S U\left(N_{c}\right)$ structure constants $f_{a b c}$. With the given color permittivity, it is possible to further analyze the non-Abelian response of QGP to external currents 12.

As an application of the nonlinear color permittivity (4.13), we can obtain the nonlinear Landau damping rate 21]

$$
\gamma=-\frac{\operatorname{Im}\left(\varepsilon^{*}\right)}{\left.\frac{\partial \varepsilon_{L}}{\partial \omega}\right|_{\omega=\omega_{\mathbf{k}}}}=0.17 g^{2} T,
$$

and the nonlinear eigenfrequency shift

$$
\Delta \omega=-\frac{R e\left(\varepsilon^{*}\right)}{\left.\frac{\partial \varepsilon_{L}}{\partial \omega}\right|_{\omega=\omega_{\mathbf{k}}}}=0.83 g^{2} T,
$$

which characterizes the nonlinear effect in QGP too. 


\section{ACKNOWLEDGMENTS}

The work was supported in part by the NSFC under Grant No. 19925519, 10135030 and 10175026. 


\section{REFERENCES}

[1] U. Heinz, Phys. Rev. Lett. 51 (1983) 351; U. Heinz, Ann. Phys. 161 (1985) 48; H.-Th. Elze and U. Heinz, Phys. Rep. 183 (1989) 81.

[2] S. Mrówczyński, Phys. Rev. D 39 (1989) 1940.

[3] J.-P. Blaizot, E. Iancu, Phys. Rev. Lett. 70 (1993) 3376; Nucl. Phys. B 421 (1994) 565.

[4] P.F. Kelly, Q. Liu, C. Lucchesi and C. Manuel, Phys. Rev. D 50 (1994) 4209.

[5] A.V. Selikhov and M. Gyulassy, Phys. Lett. B 316 (1993) 373; Phys. Rev. C 49 (1994) 1726. A.V. Selikhov, Phys. Lett. B 268 (1991) 263.

[6] D. Bödeker, Phys. Lett. B 426 (1998) 351; D. Bödeker, Nucl. Phys. B 559 (1999) 502.

[7] D.F. Litim and C. Manuel, Phys. Rev. Lett. 82 (1999) 4981; Nucl. Phys. B 562 (1999) 237.

[8] P. Arnold, D. Son and L.G. Yaffe, Phys. Rev. D 59 (1999) 105020; P. Arnold, D. Son and L.G. Yaffe, Phys. Rev. D 60 (1999) 025007.

[9] J.P. Blaizot and E. Iancu, Nucl. Phys. B 557 (1999) 183; Nucl. Phys. B 570 (2000) 326-358.

[10] M. Gyulassy and X.-N. Wang, Nucl. Phys. B 420 (1994) 583; R. Baier, Yu.L. Dokshitzer, A.H. Mueller and D. Schiff, Nucl. Phys. B 531 (1998) 403.

[11] X.-N. Wang, M. Gyulassy and M. Plümer, Phys. Rev. D 51 (1995) 3436; X.-N. Wang, Phys. Rev. Lett. 81 (1998) 2655; Phys. Rev. C 58 (1998) 2321.

[12] Y. Koike and T. Matsui, Phsy. Rev. D 45 (1992) 3237.

[13] Y.A. Markov and R.A. Markova, Transp. Theory Statist. Phys. 28 (1999) 645.

[14] Xiao-ping Zheng and Jia-rong Li, Phys. Lett. B 409 (1997) 45.

[15] Xiao-fei Zhang and Jia-rong Li, Phys. Rev. C 52 (1995) 964;

[16] Ji-sheng Chen and Jia-rong Li, Mod. Phys. Lett. A15, 1843-1849 (2000).

[17] Ji-sheng Chen and Jia-rong Li, Phys. Lett. B 430 (1998) 209; Commun. Theor. Phys., 34, 251-260 (2000); Ji-sheng Chen, Xiao-ping Zheng and Jia-rong Li, Chin. Phys. Lett., 17, 714-716 (2000).

[18] R.D. Pisarski, Phys. Rev. Lett. 68 (1992) 2891.

[19] E. Braaten and R.D. Pisarski, Nucl. Phys. B 337 (1990) 569.

[20] Xiao-fei Zhang and Jia-rong Li, Ann. Phys.(N.Y.) 250 (1996) 433.

[21] B.B. Kadomtsev, Plasma Turbulence (Academic Press, New York, 1965).

[22] E. Lifshitz and L. Pitaevskii, Physical Kinetics (Pergamon Press, Oxford, 1981).

[23] A.G. Sitenko, Fluctuations and Nonlinear Wave Interactions in Plasmas (Pergamon, Oxford, 1990). 